\title{
TAK-063, a PDEI0A Inhibitor with Balanced Activation of Direct and Indirect Pathways, Provides Potent Antipsychotic-Like Effects in Multiple Paradigms
}

\author{
Kazunori Suzuki', Akina Harada', Hirobumi Suzuki', Maki Miyamoto ${ }^{1,2}$ and Haruhide Kimura ${ }^{*, 1}$ \\ 'CNS Drug Discovery Unit, Pharmaceutical Research Division, Takeda Pharmaceutical Company Limited, Fujisawa, Japan; ${ }^{2}$ Drug Metabolism and \\ Pharmacokinetics Research Laboratories, Pharmaceutical Research Division, Takeda Pharmaceutical Company Limited, Fujisawa, Japan
}

\begin{abstract}
Phosphodiesterase IOA (PDEIOA) inhibitors are expected to be novel drugs for schizophrenia through activation of both direct and indirect pathway medium spiny neurons. However, excess activation of the direct pathway by a dopamine D, receptor agonist SKF82958 canceled antipsychotic-like effects of a dopamine $D_{2}$ receptor antagonist haloperidol in methamphetamine (METH)-induced hyperactivity in rats. Thus, balanced activation of these pathways may be critical for PDEIOA inhibitors. Current antipsychotics and the novel PDEIOA inhibitor TAK-063, but not the selective PDEIOA inhibitor MP-10, produced dose-dependent antipsychotic-like effects in METH-induced hyperactivity and prepulse inhibition in rodents. TAK-063 and MP- 10 activated the indirect pathway to a similar extent; however, MP- 10 caused greater activation of the direct pathway than did TAK-063. Interestingly, the off-rate of TAK-063 from PDE I0A in rat brain sections was faster than that of MP-10, and a slower off-rate PDEIOA inhibitor with TAK-063-like chemical structure showed an MP-10-like pharmacological profile. In general, faster off-rate enzyme inhibitors are more sensitive than slower off-rate inhibitors to binding inhibition by enzyme substrates. As expected, TAK-063 was more sensitive than MP-I0 to binding inhibition by cyclic nucleotides. Moreover, an immunohistochemistry study suggested that cyclic adenosine monophosphate levels in the direct pathway were higher than those in the indirect pathway. These data can explain why TAK-063 showed partial activation of the direct pathway compared with MP- I0. The findings presented here suggest that TAK-063's antipsychotic-like efficacy may be attributable to its unique pharmacological properties, resulting in balanced activation of the direct and indirect striatal pathways.

Neuropsychopharmacology (2016) 41, 2252-2262; doi: I0.1038/npp.2016.20; published online 24 February 2016
\end{abstract}

\section{INTRODUCTION}

Dopamine $\mathrm{D}_{2}$ receptor antagonism or partial agonism is the fundamental mechanism of action of current antipsychotics (Kapur and Mamo, 2003; Kapur et al, 1996; Nordstrom et al, 1993; Pani et al, 2007). $\mathrm{D}_{2}$ receptors are predominantly expressed in indirect pathway medium spiny neurons (MSNs) in the striatum and are coupled with an inhibitory G protein $\left(G_{i}\right)$ that impedes the enzyme activity of adenylate cyclase. Adenylate cyclase synthesizes cyclic AMP (cAMP) from ATP (Stoof and Kebabian, 1981); thus, activation of the indirect pathway by the blockade of $\mathrm{D}_{2}$ receptors and concomitant upregulation of cAMP level is thought to be a common mechanism of action of current antipsychotics.

Phosphodiesterase 10A (PDE10A) is a dual-substrate PDE that hydrolyzes both cAMP and cyclic GMP (cGMP), and is selectively expressed in MSNs (Nishi et al, 2008; Sano et al, 2008; Xie et al, 2006). Given the high level of expression of

*Correspondence: Dr H Kimura, Pharmaceutical Research Division, Takeda Pharmaceutical Company Limited, 26-I, Muraoka-higashi 2-chome, Fujisawa, Kanagawa 25I-8555, Japan, Tel: +8I 46632I859, Fax: +81 466294468, E-mail: haruhide.kimura@takeda.com

Received 5 November 2015; revised 13 January 2016; accepted I February 2016; accepted article preview online 5 February 2016
PDE10A in MSNs, PDE10A inhibitors are thought to increase cyclic nucleotide levels and activate downstream signal transduction in the striatum, similar to $\mathrm{D}_{2}$ antagonists in indirect pathway neurons. PDE10A inhibitors have also shown a promising pharmacological profile in rodents as therapeutic drugs for schizophrenia (Grauer et al, 2009; Megens et al, 2014; Schmidt et al, 2008; Smith et al, 2013). However, in a 4-week phase 2a proof-of-concept trial, Pfizer's PDE10A inhibitor MP-10 (PF-02545920) was not superior to placebo in patients with an acute exacerbation of their symptoms of schizophrenia (DeMartinis et al, 2012). Understanding the difference between MP-10 and antipsychotics is critical. Amphetamine- or methamphetamine (METH)-induced hyperactivity is widely used as a model for acute psychosis based on the dopaminergic hyperfunction hypothesis of schizophrenia (Jones et al, 2011). Antipsychotics have been reported to show dose-dependent efficacy in this paradigm; however, MP-10 exhibited a U-shaped dose-response. Disruption of prepulse inhibition (PPI) has been used in an animal model of schizophrenia-like sensorimotor gating deficits (Geyer et al, 2001; Ouagazzal et al, 2001). Unlike $D_{2}$ antagonists, MP-10 up to $30 \mathrm{mg} / \mathrm{kg}$ i.p. did not significantly increase PPI in C57BL/6J mice, although it showed significant upregulation of cyclic nucleotide levels and their downstream signaling at $3 \mathrm{mg} / \mathrm{kg}$ i.p. (Grauer et al, 2009). 
In line with this observation, the PDE10A inhibitor TP-10 with chemical structure similar to that of MP-10 also failed to improve PPI deficits in this strain (Schmidt et al, 2008). During assessment of expression changes in pathway-specific markers, PDE10A inhibition resulted in activation of both direct and indirect pathways (Grauer et al, 2009; Suzuki et al, 2015). The combined enhancement of both $D_{1}$ receptor expressing the direct pathway and $\mathrm{D}_{2}$ receptor expressing the indirect pathway could contribute to behavioral effects that differ from those of current antipsychotics.

We searched selective PDE10A inhibitors with dosedependent efficacy in both METH-induced hyperactivity in rats and PPI deficits in mice and discovered the novel and highly selective PDE10A inhibitor TAK-063 (Harada et al, 2015a; Kunitomo et al, 2014). TAK-063 has some pharmacological effects in common with MP-10 (Grauer et al, 2009; Suzuki et al, 2015): increased cAMP and cGMP levels in the mouse striatum and reduced potential for induction of cataleptic responses compared with current antipsychotics. Using the same experimental protocols, we confirmed that TAK-063, but not MP-10, produced significant antipsychotic-like effects in METH-induced hyperactivity in rats and mice and PPI deficits in mice. We further investigated the difference between these 2 compounds and found that TAK-063 exhibited a faster off-rate than MP-10. We hypothesized that PDE10A inhibitors with slower offrates may cause excessive activation of the direct pathway, which could cancel the pharmacological effects of PDE10A inhibition in the indirect pathway. Our results provide evidence that the off-rate of PDE10A inhibitors may be a critical factor in avoiding excessive activation of the direct pathway. TAK-063, with a faster off-rate and partial activation of the direct pathway, could provide a unique opportunity for further evaluation of PDE10A inhibition as a therapeutic strategy for schizophrenia.

\section{MATERIALS AND METHODS}

\section{Animals}

Male imprinting control region (ICR) and C57BL/6J mice were supplied by CLEA Japan, Inc. (Tokyo, Japan), and Sprague-Dawley (SD) rats were supplied by Charles River Laboratories Japan, Inc. (Yokohama, Japan). Homozygous Pde10a-knockout mice and their wild-type littermates were purchased from Taconic Biosciences, Inc. (Hudson, NY), and backcrossed to $\mathrm{C} 57 \mathrm{BL} / 6 \mathrm{~J}$ background. The animals were housed in a light-controlled room (12-h light/dark cycle, with lights on at 0700 hours). After an acclimation period of at least 1 week, 6- to 9-week-old animals were used. The care and use of the animals and the experimental protocols were approved by the Experimental Animal Care and Use Committee of Takeda Pharmaceutical Company Limited.

\section{Chemicals and Radioligands}

TAK-063 (1-[2-fluoro-4-(1H-pyrazol-1-yl)phenyl]-5-methoxy-3-(1-phenyl-1H-pyrazol-5-yl)pyridazin-4(1H)-one), MP-10 succinate (MP-10) (2-[4-(1-methyl-4-pyridin-4-yl-1H-pyrazol-3yl)-phenoxymethy]-quinoline succinate), compound 1 (1-[cyclopropylmethyl]-4-fluoro-5-[5-methoxy-4-oxo-3-(1-phenyl-1Hpyrazol-5-yl)pyridazin-1(4H)-yl]-3,3-dimethyl-1,3-dihydro-2H- indol-2-one], and T-773 (1-[2-fluoro-4-(tetrahydro-2H-pyran-4-yl)phenyl]-5-methoxy-3-(1-phenyl-1H-pyrazol-5-yl)pyridazin-4(1H)-one) were synthesized by Takeda Pharmaceutical Company Limited (Kunitomo et al, 2014; Yoshikawa et al, 2015). Compound 1 was referred to as compound 19e in Yoshikawa et al (2015). MP-10 has been reported as a potent and selective PDE10A inhibitor developed by Pfizer, Inc. (Grauer et al, 2009; Verhoest et al, 2009). [ $\left.{ }^{3} \mathrm{H}\right] \mathrm{T}-773$ $(37.0 \mathrm{MBq} / \mathrm{ml}$ in ethanol; specific radioactivity = $555 \mathrm{GBq} / \mathrm{mmol}$; radiochemical purity $=99.9 \%$ ) was synthesized by Quotient Bioresearch (Radiochemicals) Ltd (Cambridgeshire, UK). Additional information is described in the Supplementary Information.

\section{Drug Administration}

Drug treatment was generally performed as previously described (Suzuki et al, 2015). Methods are described in the Supplementary Information.

\section{Measurement of Cyclic Nucleotide Concentrations in the Striatum}

Cyclic nucleotide concentrations in the striatum of male ICR mice or SD rats were measured using enzyme immunoassay kits (Cayman Chemical Company, Ann Arbor, MI) as previously described (Suzuki et al, 2015).

\section{In Vivo Occupancy Study of PDE10A Inhibitors in Rodents}

PDE10A occupancies by PDE10A inhibitors were measured using non-radiolabeled T-773 as a tracer (Harada et al, 2015a). Methods are described in the Supplementary Information.

\section{Behavioral Testing}

Antipsychotic-like effects of test compounds in METH- or MK-801-induced hyperactivity in rats and mice were assessed as previously described (Suzuki et al, 2015). For the assessment of PPI of acoustic startle in mice, 8 SR-LAB acoustic startle chambers (San Diego Instruments, Inc., San Diego, CA) were used. Methods are described in the Supplementary Information.

\section{Real-Time Quantitative PCR Expression Analysis}

mRNA expression levels of substance $\mathrm{P}$ and enkephalin in the rat striatum were measured by quantitative PCR analysis as previously described (Suzuki et al, 2015). Methods are described in the Supplementary Information.

\section{In Vivo Microdialysis Study}

Microdialysis study was performed using freely moving animals implanted with a microdialysis probe (Eicom, Kyoto, Japan) in the striatum. Methods are described in the Supplementary Information. 
Table I PDEIOA Occupancy, Striatal cAMP Increase, and Suppression of MK-80I-Induced Hyperactivity by TAK-063 and MP- 10 in Mice and Rats

\begin{tabular}{|c|c|c|c|c|c|c|c|c|c|c|c|}
\hline \multirow[b]{2}{*}{ Dose (mg/kg p.o.) } & \multicolumn{5}{|c|}{ TAK-063 } & \multicolumn{6}{|c|}{ MP-10 } \\
\hline & 0.1 & 0.3 & $\mathbf{I}$ & 3 & 10 & 0.3 & I & 3 & 10 & 30 & 100 \\
\hline Striatal occupancy (\%) ${ }^{\mathrm{a}}$ & 17 & 38 & 67 & 86 & 95 & 18 & 42 & 68 & 88 & 96 & 99 \\
\hline Striatal cAMP (\% increase $)^{b}$ & $9 \pm 2$ & $34 \pm 6$ & $51 \pm 6$ & N.D. & N.D. & N.D. & $17 \pm 6$ & $23 \pm 4$ & $44 \pm 7$ & $55 \pm 2$ & N.D. \\
\hline \multicolumn{12}{|l|}{ Rat } \\
\hline Striatal occupancy (\%) ${ }^{\mathrm{a}}$ & 10 & 26 & 53 & 77 & 92 & 5 & 16 & 36 & 65 & 85 & 95 \\
\hline Striatal cAMP (\% increase $)^{d}$ & N.D. & $9 \pm 5$ & $16 \pm 4$ & $23 \pm 8$ & $31 \pm 6$ & N.D. & N.D. & $7 \pm 7$ & $39 \pm 6$ & $67 \pm 12$ & $68 \pm 15$ \\
\hline MK-80I hyperactivity (\% inhibition) ${ }^{c}$ & $14 \pm 6$ & $85 \pm 5$ & $99 \pm 2$ & N.D. & N.D. & N.D. & $27 \pm 6$ & $95 \pm 5$ & $108 \pm 1$ & N.D. & N.D. \\
\hline
\end{tabular}

Abbreviation: N.D., not determined. See also Supplementary Figure SI.

${ }^{\text {aS }}$ triatal PDEIOA occupancy was determined by regression analysis in an in vivo occupancy study using T-773 (Supplementary Figure SI) (Harada et al, 20 I5a).

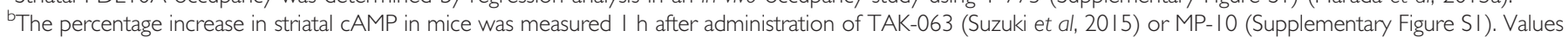
are indicated as mean \pm SEM $(n=7-8)$

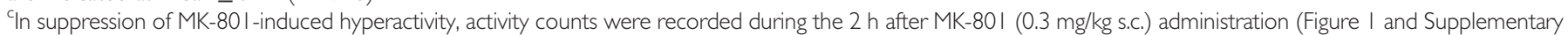
Figure SI) (Suzuki et al, 20I5). Values are indicated as mean \pm SEM $(n=5-6)$.


mean $\pm \operatorname{SEM}(n=7)$.

\section{In Vitro PDE Inhibition Assay}

Inhibitory activities of test compounds for recombinant human PDEs (1-11) were measured by scintillation proximity assay (PerkinElmer, Inc., Waltham, MA) as previously described (Kunitomo et al, 2014). Methods are described in the Supplementary Information.

\section{Autoradiography Study Using Rat Brain Sections}

Methods are described in the Supplementary Information.

\section{Immunohistochemistry}

Methods are described in the Supplementary Information.

\section{Statistical Analysis}

Statistical methods were described in the figure legends. Detailed information is described in the Supplementary Information.

\section{RESULTS}

TAK-063, but not MP-10, Produced Significant and Dose-Dependent Antipsychotic-Like Effects in METHInduced Hyperactivity in Rats and Mice and PPI Deficits in Mice

The effects of TAK-063 on behavior were evaluated in rodent models of schizophrenia and compared with that of MP-10. In rats treated with the $N$-methyl-D-aspartic acid receptor antagonist MK-801, an animal model of psychosis, both TAK-063 $(0.3 \mathrm{mg} / \mathrm{kg})$ and MP-10 $(3 \mathrm{mg} / \mathrm{kg})$ produced $>70 \%$ suppression of MK-801-induced hyperactivity at doses that resulted in 26 and 36\% PDE10A occupancy, respectively (Table 1, Figure 1a, and Supplementary Figure S1) (Harada et al, 2015a; Suzuki et al, 2015). The same results were replicated in mice, whereby treatment with either TAK-063 $(0.3 \mathrm{mg} / \mathrm{kg}, 38 \%$ occupancy $)$ or MP-10 (3 $\mathrm{mg} / \mathrm{kg}, 68 \%$ occupancy) produced $>70 \%$ reduction in MK-801-induced hyperactivity (Table 1 and Supplementary Figure S1; Suzuki et al, 2015). At efficacious dosages of reduction in MK-801-induced hyperactivity, both TAK-063 and MP-10 produced $<10 \%$ increase in striatal cAMP levels in rats, while they produced $>20 \%$ increase in cAMP levels in mice (Table 1). The antipsychotic-like efficacy of TAK-063 was further explored in METH-induced hyperactivity models of psychosis. TAK-063 produced a dose-dependent suppression of METH-induced hyperactivity in rats at $\geqslant 26 \%$ occupancies, while MP-10 had no effect $(P \leqslant 0.05$; Figure 1a). Current antipsychotics such as haloperidol, olanzapine, risperidone, and aripiprazole demonstrated dose-dependent suppression of METH-induced hyperactivity $(P \leqslant 0.05$; Supplementary Figure $S 2)$. TAK-063, but not MP-10, also produced a significant suppression of METHinduced hyperactivity in ICR mice $(P \leqslant 0.05$; Supplementary Figure S2).

C57BL/6J mice have been known to exhibit a naturally occurring deficit in PPI (Paylor and Crawley, 1997). The percentage of PPI in C57BL/6J mice was significantly lower than that in ICR mice $(P \leqslant 0.01$; Supplementary Figure S3). TAK-063 at 38,67 , and $86 \%$ occupancies $(0.3$, 1 , and $3 \mathrm{mg} / \mathrm{kg}$, respectively) significantly increased PPI in C57BL/6J mice $(P \leqslant 0.05)$, whereas MP-10 up to $96 \%$ occupancy did not show a significant effect $(30 \mathrm{mg} / \mathrm{kg}$; Figure 1b). We next used a pharmacologically manipulated PPI deficit model relevant for schizophrenia. MK-801 significantly reduced the percentage of PPI in ICR mice $(P \leqslant 0.01 ; \quad$ Figure 1c). Again, TAK-063 significantly increased the percentage of PPI in MK-801-treated mice at 38 and $67 \%$ occupancies $(0.3$ and $1 \mathrm{mg} / \mathrm{kg}$, respectively; $P \leqslant 0.05)$, whereas MP-10 did not show a significant effect up to $96 \%$ occupancy $(30 \mathrm{mg} / \mathrm{kg}$; Figure $1 \mathrm{c})$. The current antipsychotic risperidone also significantly increased the 
a

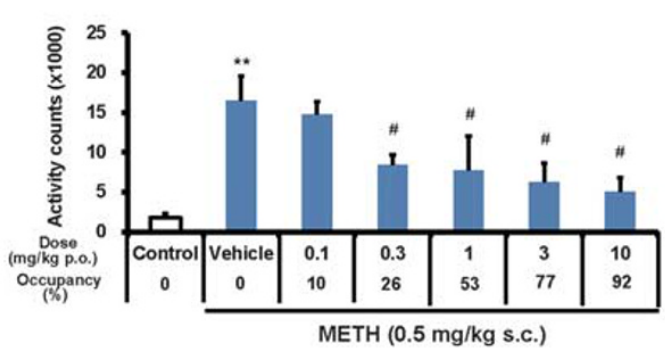

b

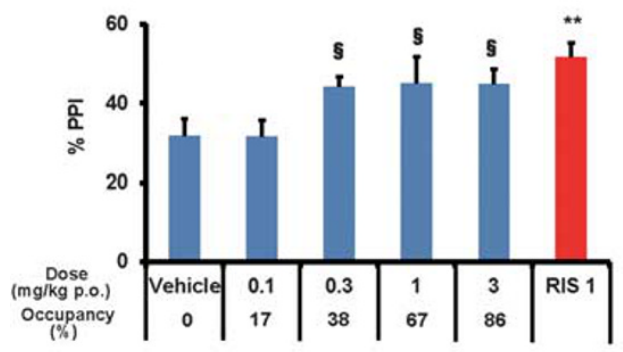

C

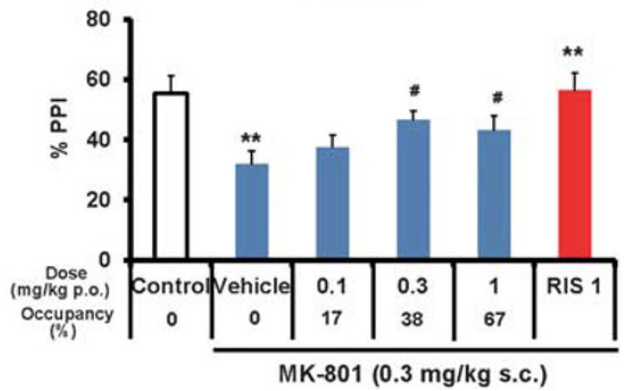

MP-10


MP-10

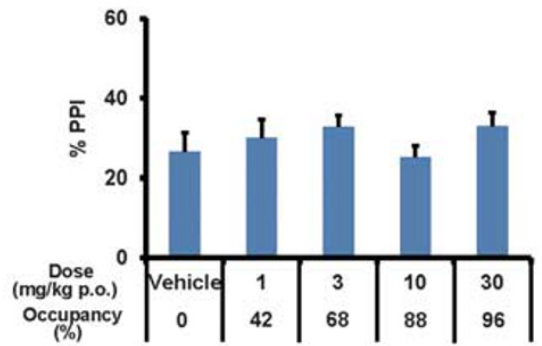

MP-10

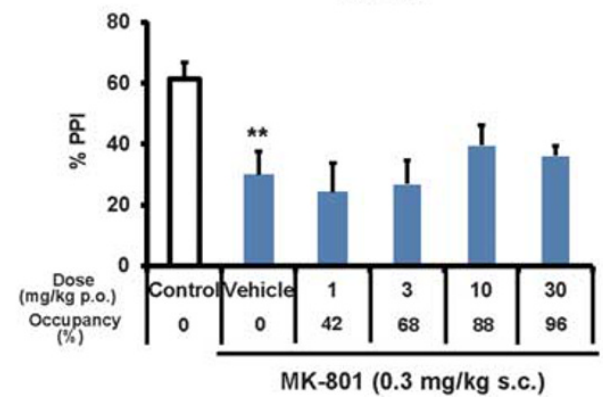

Figure I TAK-063, but not MP-10, produced significant and dose-dependent antipsychotic-like effects in METH-induced hyperactivity and PPI deficits. (a) Suppression of METH or MK-80 I-induced hyperactivity in rats. METH $(0.5 \mathrm{mg} / \mathrm{kg})$ or MK-80 I $(0.3 \mathrm{mg} / \mathrm{kg})$ was administered to rats subcutaneously I.5 or $0.5 \mathrm{~h}$ after oral administration of TAK-063 or MP-10, respectively. Accumulated activity counts during the $2 \mathrm{~h}$ after METH or MK-80I treatment were calculated and are indicated as mean+SEM $(n=4-6)$. PDEI OA occupancy at each dose used is shown below the dose. *** $P \leqslant 0.05$ vs control by Aspin-Welch test. ${ }^{\#} P \leqslant 0.05$ vs the vehicle+METH group by two-tailed Williams' test (Williams, 197 I). ${ }^{\S} P \leqslant 0.05$ vs vehicle by two-tailed Shirley-Williams test (Shirley, 1977). (b) The effects of TAK-063 and MP-I0 on PPI in C57BL/6J mice. Risperidone (RIS I, I mg/kg) was used as a control. PDEIOA occupancy at each doses used is shown below the dose. Data (\%PPI to the $82-\mathrm{dB}$ prepulse) are presented as mean+SEM. $(n=10-13)$. ${ }^{\S} P \leqslant 0.05$ vs vehicle by two-tailed Shirley-Williams test. $* * * 0.01$ vs vehicle by Aspin-Welch test. (c) The effect of TAK-063 and MP-I0 on MK-80I-induced PPI deficits in ICR mice. Risperidone (RIS I, I mg/kg) was used as a control. PDE IOA occupancy at each dose used is shown below the dose. Data (\%PPI to the 82-dB prepulse) are presented as mean+SEM $(n=8-16)$. ${ }^{*} * P \leqslant 0.01$ vs vehicle by Aspin-Welch test. ${ }^{\#} P \leqslant 0.05$ vs vehicle by two-tailed Williams' test. See also Supplementary Figures S2 and S3. METH, methamphetamine; ICR, imprinting control region; PPI, prepulse inhibition.

percentage of PPI in both tests $(P \leqslant 0.01$; Figure $1 \mathrm{~b}$ and $\mathrm{c})$. These results suggest that TAK-063, but not MP-10, has antipsychotic-like effects in METH-induced hyperactivity and PPI deficits at doses relevant for suppression of MK-801induced hyperactivity.

\section{Activation of the Direct Pathway by MP-10 was Greater than Activation by TAK-063}

PDE10A inhibitors are known to activate both direct and indirect pathways (Grauer et al, 2009). These pathways are considered to have competing effects on striatal outputs (DeLong, 1990; Obeso et al, 2004). In fact, both the cataleptic (Suzuki et al, 2015) and the antipsychotic-like effects of haloperidol, a $\mathrm{D}_{2}$-receptor antagonist, were canceled by co-administration of the $\mathrm{D}_{1}$ agonist SKF82958 $(P \leqslant 0.05$; Figure 2a). Thus, understanding of activation pattern of these pathways caused by PDE10A inhibitors is critical. We evaluated the activation of these pathways in the rat striatum by using induction of genes as pathway-specific markers: enkephalin for the indirect pathway and substance $\mathrm{P}$ for the direct pathway (Gerfen et al, 1990; Simpson and McGinty, 1995). TAK-063 and MP-10 dose-dependently increased enkephalin mRNA to similar levels: $56 \%$ increase at $92 \%$ occupancy of TAK-063 $(10 \mathrm{mg} / \mathrm{kg})$ and $59 \%$ increase at $95 \%$ occupancy of MP-10 (100 mg/kg; Figure 2b). In contrast, MP-10 induced a greater increase in substance P mRNA than did TAK-063; MP-10 induced a $114 \%$ increase at $85 \%$ occupancy $(30 \mathrm{mg} / \mathrm{kg})$ and a $144 \%$ increase at $95 \%$ occupancy $(100 \mathrm{mg} / \mathrm{kg})$, whereas TAK-063 induced only a $76 \%$ increase 
at $92 \%$ occupancy $(10 \mathrm{mg} / \mathrm{kg}$; Figure $2 \mathrm{~b})$. Calculation of the ratio of substance $\mathrm{P}$ mRNA to enkephalin mRNA after administration of these compounds (Figure 2c) revealed that MP-10, but not TAK-063, induced a significant increase in the relative expression of substance $\mathrm{P}$ (substance $\mathrm{P} / \mathrm{enkephalin}$, $P \leqslant 0.05$ ) at 85 and $95 \%$ occupancies $(30$ and $100 \mathrm{mg} / \mathrm{kg}$, respectively). This result suggests that TAK-063 and MP-10 activated the indirect pathway to a similar extent, although, at $\geqslant 85 \%$ occupancy, MP-10 induced greater activation of the direct pathway than did TAK-063.

\section{MP-10, but not TAK-063, Markedly Induced Dopamine Release in the Striatum}

Several studies have reported that activation of the direct pathway is expected to cause striatal dopamine release from the substantia nigra pars compacta (Grace and Bunney, 1985; Mailly et al, 2003; Walters and Howard, 1990). We confirmed that the $\mathrm{D}_{1}$ agonist SKF82958 induced dopamine release in the rat striatum $(P \leqslant 0.01$, at $60 \mathrm{~min}$ after injection; Figure $2 \mathrm{~d}$ ). This result suggests that activation of the direct pathway by PDE10A inhibitors also induces striatal dopamine release. Repeated measures analysis of variance (RMANOVA) showed that while treatment with MP-10 at 85 and 95 occupancies (30 and $100 \mathrm{mg} / \mathrm{kg}$, respectively) results in a significant increase in striatal dopamine release, as evidenced by a time-by-treatment interaction, TAK-063 administration is not associated with significant increases in striatal dopamine even at higher occupancies (92\%, corresponding to $0.3-10 \mathrm{mg} / \mathrm{kg}$ ). Post hoc analysis confirmed significant increases in striatal dopamine release at 120, 140, and $180 \mathrm{~min}$ following MP-10 administration $(P \leqslant 0.025$; Figure 2e). Pde10a-knockout mice were used to confirm the role of PDE10A in TAK-063- and MP-10-dependent dopamine release in the striatum. In Pde10a-knockout mice, TAK-063 does not accumulate in the striatum (Harada et al, 2015a) and is not effective in reversing phencyclidine-induced hyperactivity (Kunitomo et al, 2014). The slight dopamine release by TAK-063 at $86 \%$ occupancy ( $3 \mathrm{mg} / \mathrm{kg}$ ) was absent in Pde10a-knockout mice and the excessive dopamine release by MP-10 at $96 \%$ occupancy $(30 \mathrm{mg} / \mathrm{kg})$ was also completely abolished in Pde10a-knockout mice; thus, PDE10A inhibition appears to induce dopamine release (Figure 2f). It is known that $\mathrm{METH}$ induces hyperactivity through upregulation of dopamine levels in rodents (Fukushima et al, 2007). MP-10 at $\geqslant 85 \%$ occupancy may lose its antipsychotic-like effects by excessive activation of the direct pathway and dopamine release.

\section{Off-Rate From PDE10A May Characterize Activation of Direct and Indirect Pathways and Pharmacological Profiles of PDE10A Inhibitors}

To gain insight into the difference between TAK-063 and MP-10, we assessed the binding kinetics of these compounds using the PDE10A-selective radioligand $\left[{ }^{3} \mathrm{H}\right] \mathrm{T}-773$ as a tracer (Harada et al, 2015b). In previous studies, we observed that binding of $\left[{ }^{3} \mathrm{H}\right] \mathrm{T}-773$ to PDE10A in rat brain sections was competitively inhibited by TAK-063 and MP-10 (Harada et al, 2015a; Harada et al, 2015b). The off-rates of TAK-063 and MP-10 from PDE10A in rat brain sections were measured on the basis of the reduction speed of PDE10A occupancy by these compounds (Figure 3a) (Haddad et al, 1994). The PDE10A occupancies of TAK-063 and MP-10 at 60 -min incubation after saturation were $23.09 \pm 1.90 \%$ and $49.85 \pm 1.94 \%$, respectively. MP-10 showed a slower off-rate than TAK-063 (Figure 3b). To understand more about the relationship between the off-rates and the pharmacological effects of PDE10A inhibitors, we conducted pharmacological studies using compound 1 , which has a chemical structure similar to that of TAK-063 (Yoshikawa et al, 2015). Compound 1 showed an $\mathrm{IC}_{50}$ value of $0.08 \mathrm{nM}$ for PDE10A2 inhibition and its selectivity over other PDEs was $>1800$ fold (Supplementary Table S1). A dissociation study showed that the PDE10A occupancy of compound 1 at 60 -min incubation after saturation was $43.95 \pm 1.91 \%$, indicating an off-rate similar to that of MP-10 (Figure 3b). Competition kinetics study using $\left[{ }^{3} \mathrm{H}\right] \mathrm{T}-773$ also supported that the dissociation speed of TAK-063 was faster than those of MP-10 and compound 1; the dissociation half-life of TAK-063, MP-10 and compound 1 were 8,42 ,

Figure 2 Compared with TAK-063, MP-I0 induced greater levels of direct pathway activation and dopamine release that can cancel antipsychotic effect by $\mathrm{D}_{2}$ antagonism. (a) Effect of SKF82958 on the antipsychotic-like effect of haloperidol in METH-induced hyperactivity in rats. METH (0.5 mg/kg) was administered subcutaneously I and $0.5 \mathrm{~h}$ after administration of haloperidol (HAL, $0.3 \mathrm{mg} / \mathrm{kg}$ p.o.) and SKF82958 (SKF, I, 3, and I0 mg/kg i.p.), respectively. Accumulated activity counts during the $2 \mathrm{~h}$ after METH treatment were calculated and are indicated as mean $+\mathrm{SEM}(n=5)$. $* * P \leqslant 0.0 \mathrm{I}$ between the control group and METH-treated group by Aspin-Welch test. $* P \leqslant 0.05$ between the METH-treated group and HAL+METH-treated group by Aspin-Welch test. ${ }^{\#} P \leqslant 0.05$ vs the HAL+vehicle-treated group by two-tailed Williams' test. (b) Three hours after administration of TAK-063 or MP- I0, mRNA expression levels of substance P (SP, a marker of the direct pathway) and enkephalin (Enk, a marker of the indirect pathway) in the rat striatum were measured by PCR analysis. PDEIOA occupancy at each dose used is shown below the dose. The values in the graph represent expression levels relative to that of the vehicle-treated group. Data are presented as mean+SEM $(n=6) . \S P \leqslant 0.05$ vs the vehicle-treated group by two-tailed Williams' test (for SP expression). ${ }^{\#} P \leqslant 0.05$ vs the vehicle-treated group by two-tailed Williams' test (for Enk expression). (c) Ratio of substance P mRNA to enkephalin mRNA after PDEIOA inhibition. PDEIOA occupancy at each dose used is shown below the dose. The values in the graph represent expression ratio of substance $P$ to enkephalin (SP/Enk) relative to that of the vehicle-treated group. Data are presented as mean+SEM $(n=6)$. ${ }^{*} P \leqslant 0.05$ vs the vehicle-treated group by two-tailed Williams' test. (d) Striatal dopamine release during continuous injection of SKF82958 (I00 $\mu \mathrm{M}, \mathrm{I} \mu \mathrm{l} / \mathrm{min}$ ) into the rat striatum for 60 min. SKF82958 was continuously injected from time 0. Data are expressed as the percentage of baseline dopamine levels and are mean+SEM $(n=6)$. *** $\leqslant 0.01$ between the SKF82958-injected group and vehicle-injected group at $60 \mathrm{~min}$ after the start of injection by Aspin-Welch test. (e) Striatal dopamine release by TAK-063 or MP-I0 was measured using microdialysis in rats. Drugs were administered at time 0. Data are presented as the percentage of baseline dopamine levels and are mean+SEM $(n=5-6)$. RM-ANOVA was followed by Williams' test (for parametric data, $P>0.05$ by Bartlett's test) or Shirley-Williams test (for non-parametric data, $P \leqslant 0.05$ by Bartlett's test). ${ }^{\#} P \leqslant 0.05$ vs the vehicle-treated group by two-tailed Williams' test at each time point. (f) Striatal dopamine release by TAK-063 and MP-I 0 was measured using microdialysis in Pde l Oa-knockout mice $(\mathrm{KO})$ and their wild-type littermates (WT). Drugs were administered at time 0 . Data are presented as the percentage of baseline dopamine levels and are mean+SEM $(n=3-5)$. METH, methamphetamine; RM-ANOVA, repeated measures analysis of variance. 
and $27 \mathrm{~min}$, respectively (Supplementary Figure S4 and Table S2). Unlike TAK-063, compound 1 showed an MP-10-like pharmacological profile in rodents (Figure $3 \mathrm{c}-\mathrm{g}$ and Supplementary Figure S5). These data further support the hypothesis that off-rates from PDE10A may characterize the pharmacological profiles of PDE10A inhibitors through modulation of activation level of the direct pathway.
Higher Cyclic Nucleotide Concentrations in the Direct Pathway Would Lead to a Partial PDE10A Inhibition by TAK-063 in the Direct Pathway MSNs

$\mathrm{D}_{2}$ receptors, predominantly expressed in indirect pathway MSNs, are coupled with $G_{i}$ and inhibit adenylate cyclase, whereas $D_{1}$ receptors, predominantly expressed in direct pathway MSNs, are coupled with stimulatory $G$ protein $\left(G_{s}\right)$
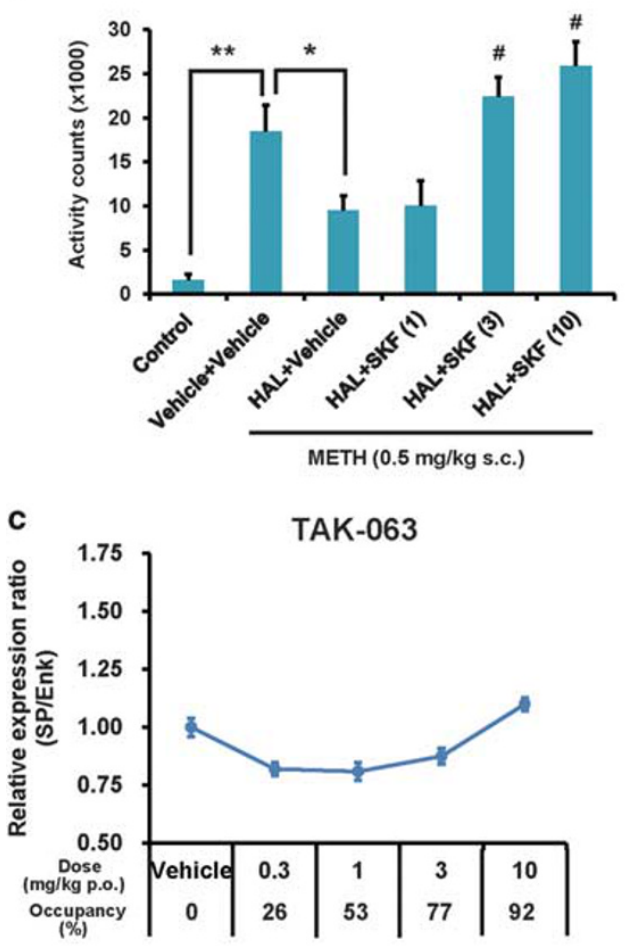

d

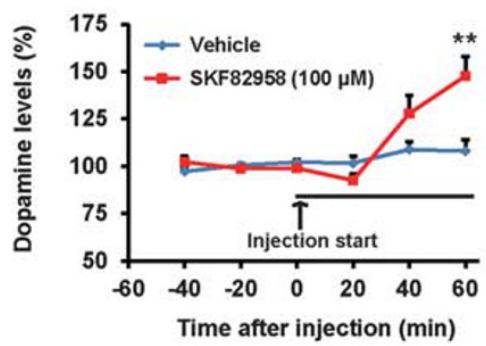

f



b



TAK-063



MP-10

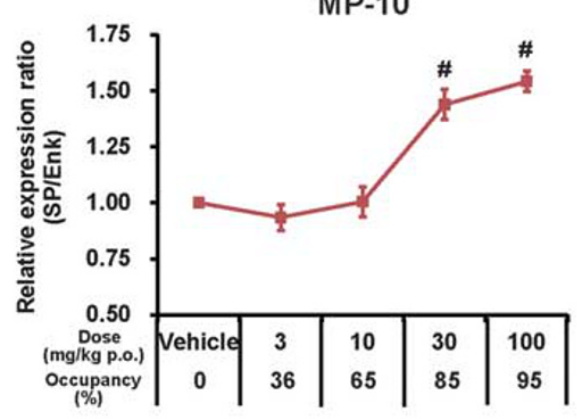

e

TAK-063

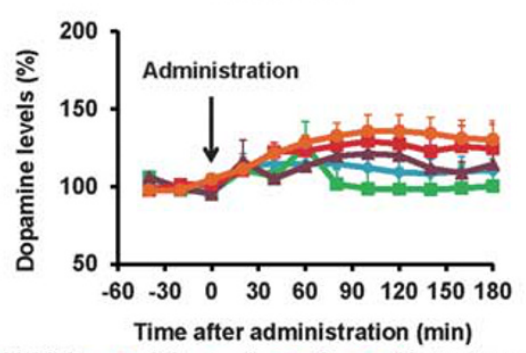

MP-10

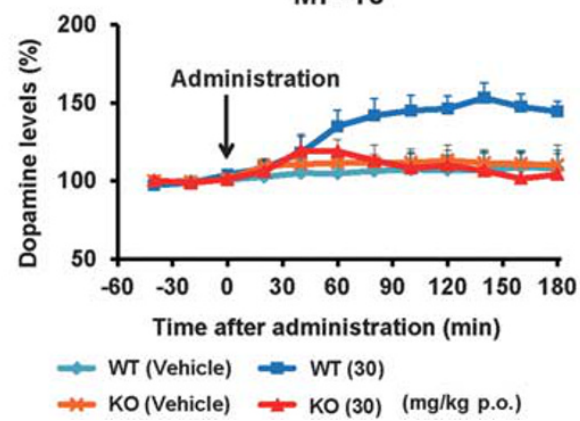

MP-10






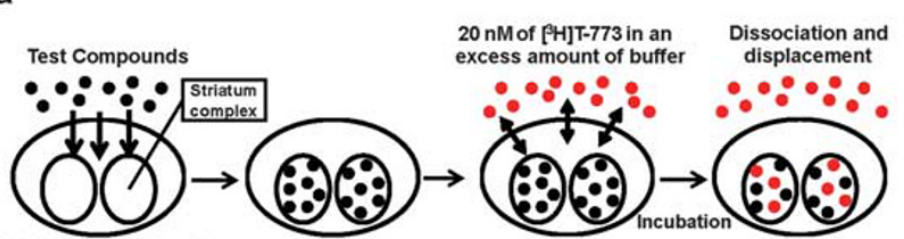

Rat brain coronal section

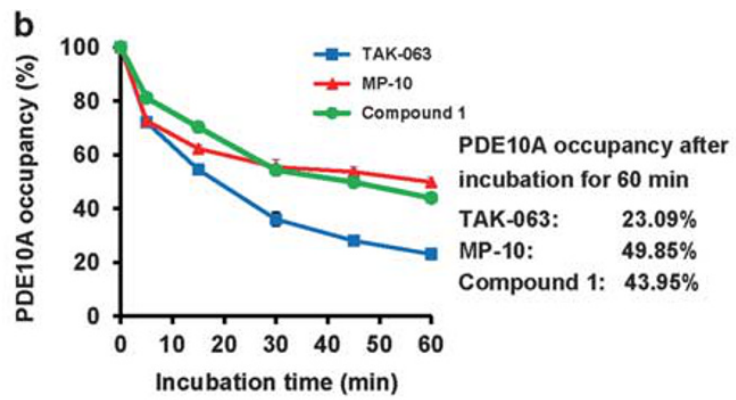

C


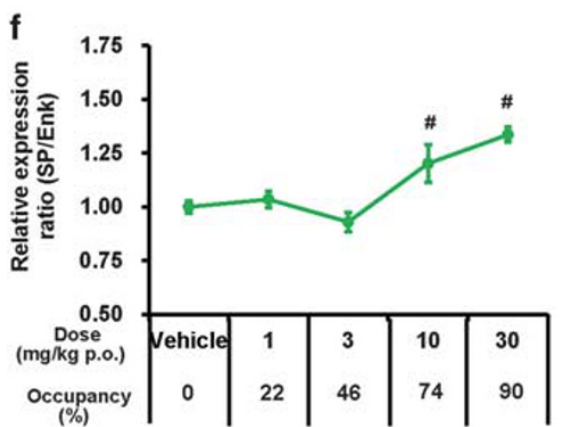

g



Figure 3 Off-rates of PDEIOA inhibitors may characterize their pharmacological profiles via regulation of each MSN pathway. (a) Schematic illustration of the experimental procedure to evaluate off-rates of PDEIOA inhibitors. The off-rates of TAK-063 and MP-I0 from PDEIOA in rat brain sections were obtained by measuring their PDE IOA occupancy using $\left[{ }^{3} \mathrm{H}\right] \mathrm{T}-773$ as a tracer. (b) Comparison of the off-rates of TAK-063, MP-I0, and compound I from PDE IOA. Data are presented as mean+SEM $(n=3)$. The PDEIOA occupancies of TAK-063, MP-I0, and compound I at 60-min incubation after saturation in rat brain sections were $23.09,49.85$, and $43.95 \%$, respectively. (c) Suppression of METH-induced hyperactivity in rats. METH was administered $3 \mathrm{~h}$ after administration of compound I. Accumulated activity counts during the $2 \mathrm{~h}$ after METH treatment were calculated. Data are presented as mean $+\mathrm{SEM}(\mathrm{n}=5)$. * $P \leqslant 0.05$ vs control by Aspin-Welch test. (d) The effects of compound I on PPI in C57BL/6) mice. Data are presented as mean+SEM ( $n=6-8)$. (e) Activation of MSNs by compound I. mRNA levels of substance P (SP) and enkephalin (Enk) in the rat striatum were measured by quantitative PCR $3 \mathrm{~h}$ after administration of compound I. Data are presented as mean+SEM $(n=6) .{ }^{\S} P \leqslant 0.05,{ }^{\#} P \leqslant 0.05$ by two-tailed Williams' test. ( $f$ ) Ratio of substance $P$ mRNA to enkephalin mRNA after PDE IOA inhibition. PDE I OA occupancy at each dose is shown below the dose. The values in the graph represent ratio of substance $P$ to enkephalin (SP/Enk) expression relative to that of the vehicle-treated group. Data are presented as mean+SEM $(n=6)$. ${ }^{\#} P \leqslant 0.05$ vs the vehicle-treated group. (g) Striatal dopamine release by compound I was measured using microdialysis in rats. Data are presented as the percentage of baseline dopamine levels $(n=6-7)$. RM-ANOVA was followed by Williams' test (for parametric data, $P>0.05$ by Bartlett's test) or Shirley-Williams test (for non-parametric data, $P \leqslant 0.05$ by Bartlett's test). ${ }^{\#} P \leqslant 0.05$ vs the vehicle-treated group by two-tailed Williams' test at each time point. ${ }^{\S} P \leqslant 0.05$ vs the vehicle-treated group by twotailed Shirley-Williams test at each time point. See also Supplementary Figure S5. METH, methamphetamine; MSN, medium spiny neuron; PPI, prepulse inhibition; RM-ANOVA, repeated measures analysis of variance.

and stimulate adenylate cyclase. Thus, cAMP levels in the direct pathway MSNs are speculated to be higher than that in the indirect pathway MSNs. In fact, an immunohistochemical analysis using the antibodies against cAMP and substance $\mathrm{P}$, a marker of the direct pathway MSNs, showed that $>90 \%$ of cAMP-positive cells were co-immunostained with the anti-substance $\mathrm{P}$ antibody in the rat striatum after treatment with either vehicle or MP-10 $(94.56 \pm 1.02 \%$ in vehicle-treated group, $95.88 \pm 0.82 \%$ in MP-10-treated group, $n=3$; Figure 4a). The main source of cAMP in MSNs is thought to be derived from the direct pathway MSNs.
Quantitative analysis of striatal cAMP and cGMP levels showed that while both MP-10 and TAK-063 dose-dependently increased cAMP and cGMP levels, treatment with MP-10 might produce larger increases in cyclic nucleotide levels (Figure 4b); MP-10 induced a 67\% increase in cAMP level at $85 \%$ occupancy $(30 \mathrm{mg} / \mathrm{kg})$, whereas TAK-063 induced a $31 \%$ increase at $92 \%$ occupancy $(10 \mathrm{mg} / \mathrm{kg}$; Figure 4b). Both TAK-063 and MP-10 bind to the substrate-binding site in the catalytic domain of PDE10A (Kunitomo et al, 2014; Verhoest et al, 2009); thus, cAMP and cGMP levels increased by PDE10A inhibition and/or 
dopamine increase can compete with TAK-063 and MP-10 at this domain. Under conditions with higher cyclic nucleotide concentrations, the binding of these compounds to PDE10A possibly differs depending on their off-rates. To address this possibility, we evaluated the binding of TAK-063 and MP-10 to PDE10A in the presence of cyclic nucleotides (cAMP and cGMP) in rat brain sections. Two-way ANOVA revealed a significant interaction effect between concentrations of cyclic nucleotides and treatment. Post hoc analysis showed significant differences between TAK-063 and MP-10 at $6 \mathrm{mM}$ (3 mM each) and $60 \mathrm{mM}$ (30 mM each) of cyclic nucleotides $(P \leqslant 0.05$ by Aspin-Welch test; Figure $4 \mathrm{c})$. TAK-063 was more sensitive than MP-10 to binding inhibition by cyclic nucleotides. Competitive binding studies revealed that the $\mathrm{Ki}$ values of these compounds were similar in rat brain sections (Figure 4d). These results suggest that MP-10 with its slower off-rate can inhibit PDE10A more potently than TAK-063 under conditions of higher cyclic nucleotide concentrations.

\section{DISCUSSION}

We searched PDE10A inhibitors with dose-dependent efficacies in both METH-induced hyperactivity in rats and PPI deficits in mice and discovered the novel and highly selective PDE10A inhibitor TAK-063 (Harada et al, 2015b; Kunitomo et al, 2014). TAK-063, but not MP-10, produced significant antipsychotic-like effects in METH-induced hyperactivity and PPI deficits (Figure 1), although both TAK-063 and MP-10 are highly selective for PDE10A.

Using binding kinetics studies, we determined that TAK-063 has a faster off-rate from PDE10A than MP-10 does (Figure 3b, Supplementary Figure S4 and Table S2). Moreover, compound 1, a PDE10A inhibitor with a chemical structure similar to that of TAK-063 and an off-rate similar to that of MP-10, showed an MP-10-like pharmacological profile (Figure 3 ). These results suggest that off-rates from PDE10A characterize the pharmacological profile of PDE10A inhibitors. PDE10A inhibitors are known to activate both direct and indirect pathways (Grauer et al, 2009), which are thought to have competing effects on striatal outputs (Figure 2) (DeLong, 1990; Obeso et al, 2004). Although TAK-063 and MP-10 activated the indirect pathway to a similar extent, at $\geqslant 85 \%$ occupancy, MP-10 induced greater activation of the direct pathway than did TAK-063 (Figure 2). This excessive activation of the direct pathway was also observed with compound 1 (Figure $3 \mathrm{e}$ and $\mathrm{f}$ ). Activation of the direct pathway by a $D_{1}$ agonist is expected to cause striatal dopamine release (Walters and Howard, 1990). In fact, the $D_{1}$ agonist SKF82958 induced dopamine release in the rat striatum (Figure 2d). In line with this observation, both MP-10 and compound 1, but not TAK-063 , induced significant dopamine release in the rat striatum, whereas MP-10-induced dopamine release was absent in Pde10a-knockout mice (Figures 2 and 3). It is known that METH induces hyperactivity through upregulation of dopamine levels in rodents (Fukushima et al, 2007). We concluded that PDE10A inhibitors with slower off-rates may cause excessive activation of the direct pathway and that this activation, at least partially facilitated through dopamine release, can cancel their antipsychotic effects via PDE10A inhibition in the indirect pathway.
Compared with METH-induced hyperactivity, more robust activation of the direct pathway by a higher dose of the $\mathrm{D}_{1}$ agonist SKF82958 $(10 \mathrm{mg} / \mathrm{kg})$ was needed to cancel an antipsychotic-like effect of haloperidol in MK-801-induced hyperactivity (Supplementary Figure S6). While further studies are needed, these results suggest that MP-10 can show significant effects in this test.

We next investigated why off-rates of PDE10A inhibitors would affect their activation patterns of the direct pathway. $D_{2}$ receptors are coupled with $G_{i}$ and inhibit adenylate cyclase, whereas $D_{1}$ receptors are coupled with $G_{s}$ and stimulate adenylate cyclase. Thus, cAMP levels in direct pathway MSNs can be higher than those in indirect pathway MSNs, particularly when dopamine levels are high. In fact, most of cAMP-positive cells were co-stained with the antisubstance $P$ antibody in the rat striatum (Figure $4 \mathrm{a}$ ). Both TAK-063 and MP-10 bind to the substrate-binding site in the catalytic domain of PDE10A (Kunitomo et al, 2014; Verhoest et al, 2009); thus, increased cAMP and cGMP by PDE10A inhibition and/or dopamine increase could compete with TAK-063 and MP-10 at this domain. In general, faster offrate enzyme inhibitors are more sensitive than slower offrate inhibitors to binding inhibition by enzyme substrates. For example, the faster off-rate dipeptidyl peptidase-4 inhibitor sitagliptin, but not the slower off-rate inhibitor saxagliptin, showed decreased inhibitory activity at higher concentrations of substrate compared with lower concentrations (Wang et al, 2012). Similarly, TAK-063 binding to PDE10A was more readily inhibited by lower concentrations of cyclic nucleotides than MP-10 (Figure 4).

A possible mechanism of how off-rates of PDE10A inhibitors characterize their pharmacological profile is shown in Supplementary Figure S7. When activation of the direct pathway reaches a critical level because of PDE10A inhibition, the released dopamine stimulates $G_{s}$-coupled $D_{1}$ receptors to produce cyclic nucleotides in this pathway. In the presence of higher concentrations of cyclic nucleotides, MP-10 could inhibit PDE10A more potently than TAK-063 does, and further activate the direct pathway via upregulated cyclic nucleotides; this in turn lead to additional dopamine release in the striatum. This synergistic upregulation of cyclic nucleotide levels could lead to excessive activation of the direct pathway, a hypothesis consistent with our results regarding the effects of TAK-063, MP-10, and compound 1. Because of competitive binding inhibition by cyclic nucleotides, faster off-rate inhibitors, such as TAK-063, may lose PDE10A inhibitory activity in the direct pathway during this cycle. Under these conditions, cyclic nucleotide levels in the indirect pathway should be lower than those in the direct pathway because $D_{2}$ receptors are coupled with the $G_{i}$. Therefore, no difference in activation of the indirect pathway would be detected between TAK-063 and MP-10.

The x-ray crystal structure of compound 1 and TAK-063 with PDE10A revealed that gem-dimethyl groups of compound 1 interact with Phe629 and Met703 and the cyclopropyl group interacts with Ala626 in addition to the putative pharmacophore of TAK-063 (Kunitomo et al, 2014; Yoshikawa et al, 2015). These interactions may contribute to a slower off-rate profile of compound 1 compared with that of TAK- 063 .

Acute studies using brain slices showed that intracellular dialysis of $1 \mathrm{mM}$ of cAMP or $0.5 \mathrm{mM}$ of exogenous applied 
a
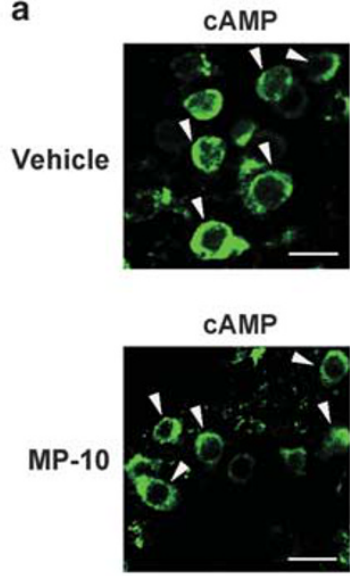

b


cAMP analogue was required to produce membrane depolarization. Moreover, the signaling pathway of cAMPdependent protein kinase in MSNs was activated by $1 \mathrm{mM}$ of cAMP analogue (Nishi et al, 1997; Podda et al, 2010). Local cyclic nucleotide levels $>6 \mathrm{mM}$ in MSNs, particularly in the
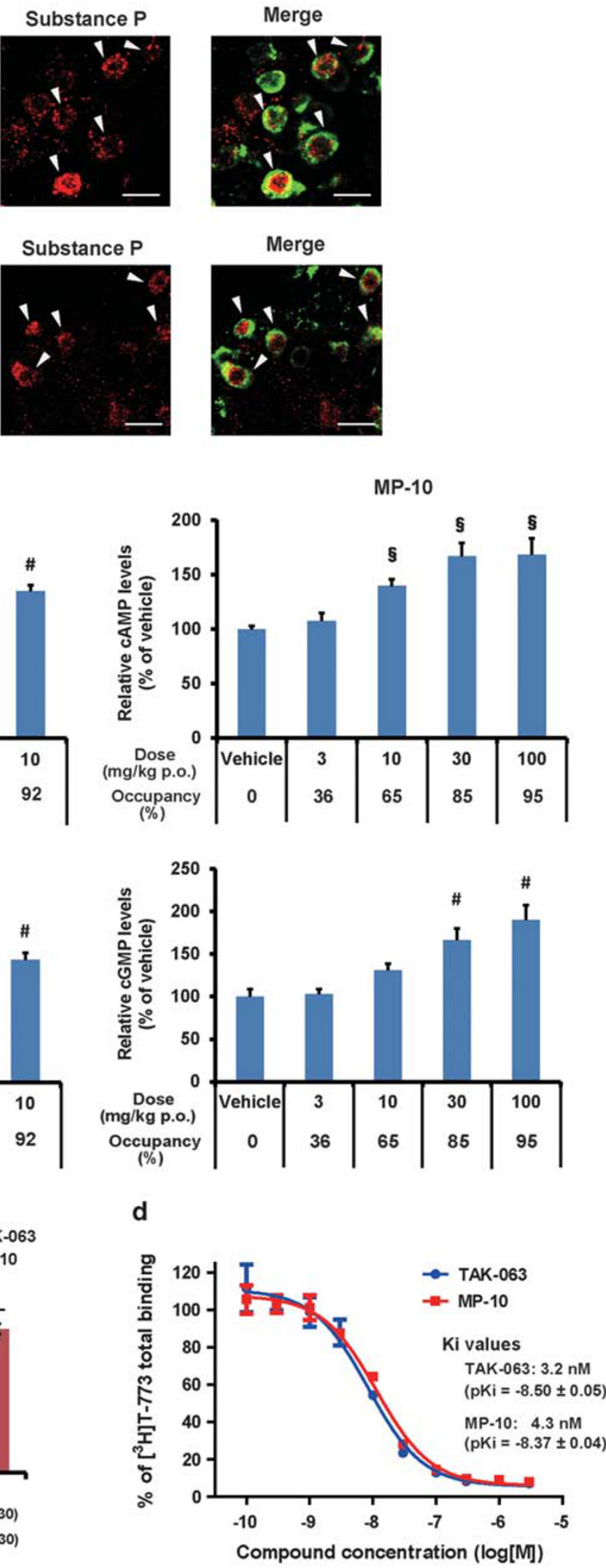

direct pathway, are plausible when PDE10A activity is inhibited, although further studies are needed.

Several reports have suggested that overactivity in the direct pathway is involved in the production of dystonia (Burbaud, 2012; Janavs and Aminoff, 1998); thus, faster off- 
Figure 4 Higher cyclic nucleotide concentrations in the direct pathway and higher sensitivity of TAK-063 for binding inhibition by cyclic nucleotides would lead to a partial PDEIOA inhibition by TAK-063 in the direct pathway MSNs. (a) Representative images of cAMP and substance P staining in coronal sections of the rat striatum at $0.5 \mathrm{~h}$ after treatment with vehicle or MP- 10 (30 mg/kg p.o.). The images were processed using NIS-Elements AR to adjust brightness and contrast. The arrowheads indicate representative cells double positive for anti-cAMP and anti-substance P staining. Scale bar, I0 $\mu \mathrm{m}$. (b) Elevation of cAMP and cGMP levels in the rat striatum at 2 and $0.5 \mathrm{~h}$ after administration of TAK-063 and MP- I0, respectively. PDE I OA occupancy at each dose used is shown below the dose. Data are presented as mean+SEM $(n=7) .{ }^{*} P \leqslant 0.05$ vs the vehicle-treated group by two-tailed Williams' test. $\S P \leqslant 0.05$ vs the vehicle-treated group by two-tailed Shirley-Williams test. (c) Binding of TAK-063 and MP-IO to PDEIOA in rat brain coronal sections in the presence or absence of cyclic nucleotides (cAMP and cGMP) at levels of $0.6 \mathrm{mM}$ ( $0.3 \mathrm{mM}$ each), $6 \mathrm{mM}$ (3 mM each), and $60 \mathrm{mM}$ (30 mM each). Data are presented as mean $\pm \mathrm{SEM}$ ( $n=4)$. The values in the graph represent binding levels relative to those in the absence of cyclic nucleotides. Data were analyzed by two-way ANOVA followed by Aspin-Welch test. $* * * \leqslant 0.01$. (d) The binding affinities of TAK-063 and MP-I0 to PDE I OA in rat coronal sections were determined by competitive binding assays using $\left[{ }^{3} \mathrm{H}\right] \mathrm{T}-773$. Data are presented as mean \pm SEM $(n=3)$. ANOVA, analysis of variance; cAMP, cyclic AMP; cGMP, cyclic GMP; MSN, medium spiny neuron.

rate PDE10A inhibitors may also have an improved pharmacological profile for tolerability.

In summary, we found that the off-rates of PDE10A inhibitors may characterize their pharmacological profiles via regulation of each MSN pathway. With its balanced activation of direct and indirect pathways via a faster off-rate profile, TAK-063 may provide a unique opportunity to validate PDE10A inhibition as a novel therapeutic approach to treating schizophrenia and is currently in clinical development (ClinicalTrials.gov Identifier: NCT02477020).

\section{Study Limitations}

In this study, we could not measure accurate cyclic nucleotide concentrations in each MSN nor cell-specific PDE10A occupancies by PDE10A inhibitors because of mosaic distribution of the indirect and direct pathway MSNs. Future work will include pathway-specific characterization of PDE10A inhibitors. Other mechanisms, such as regulation of PDE10A activity by its GAFb domain, PDE10A localization through palmitoylation, and unknown regulation through binding partners of PDE10A (Charych et al, 2010; Jäger et al, 2012; Russwurm et al, 2015), are worth evaluating.

\section{FUNDING AND DISCLOSURE}

The authors are employees of Takeda Pharmaceutical Company Limited. The authors declare no other conflict of interests.

\section{ACKNOWLEDGMENTS}

We thank the following Takeda Pharmaceutical Company Limited employees: $\mathrm{T}$ Ishii for conducting in vitro PDE enzyme assays, $M$ Harada for evaluating dopamine release by in vivo microdialysis, and $\mathrm{T}$ Taniguchi for synthesizing compounds.

\section{REFERENCES}

Burbaud P (2012). Dystonia Pathophysiology: A Critical Review. In: Raymond Rosales (ed.). Dystonia - The Many Facets. InTech: Rijeka, Croatia, pp 199-220.

Charych EI, Jiang LX, Lo F, Sullivan K, Brandon NJ (2010). Interplay of palmitoylation and phosphorylation in the trafficking and localization of phosphodiesterase 10A: implications for the treatment of schizophrenia. J Neurosci 30: 9027-9037.

DeLong MR (1990). Primate models of movement disorders of basal ganglia origin. Trends Neurosci 13: 281-285.
DeMartinis N, Banerjee A, Kumar V, Boyer S, Schmidt C, Arroyo S (2012). Results of a phase 2a proof-of-concept trial with a PDE10A inhibitor in the treatment of acute exacerbation of Schizophrenia. Schizophrenia Res 136: S262.

Fukushima S, Shen H, Hata H, Ohara A, Ohmi K, Ikeda K et al (2007). Methamphetamine-induced locomotor activity and sensitization in dopamine transporter and vesicular monoamine transporter 2 double mutant mice. Psychopharmacology (Berl) 193: 55-62.

Gerfen CR, Engber TM, Mahan LC, Susel Z, Chase TN, Monsma FJ Jr. et al (1990). D1 and D2 dopamine receptor-regulated gene expression of striatonigral and striatopallidal neurons. Science 250: 1429-1432.

Geyer MA, Krebs-Thomson K, Braff DL, Swerdlow NR (2001). Pharmacological studies of prepulse inhibition models of sensorimotor gating deficits in schizophrenia: a decade in review. Psychopharmacology (Berl) 156: 117-154.

Grace AA, Bunney BS (1985). Opposing effects of striatonigral feedback pathways on midbrain dopamine cell activity. Brain Res 333: 271-284.

Grauer SM, Pulito VL, Navarra RL, Kelly MP, Kelley C, Graf R et al (2009). Phosphodiesterase 10A inhibitor activity in preclinical models of the positive, cognitive, and negative symptoms of schizophrenia. J Pharmacol Exp Ther 331: 574-590.

Haddad EB, Mak JC, Barnes PJ (1994). Characterization of [3H]Ba 679 BR, a slowly dissociating muscarinic antagonist, in human lung: radioligand binding and autoradiographic mapping. $\mathrm{Mol}$ Pharmacol 45: 899-907.

Harada A, Suzuki K, Kamiguchi N, Miyamoto M, Tohyama K, Nakashima K et al (2015a). Characterization of binding and inhibitory properties of TAK-063, a novel phosphodiesterase 10A inhibitor. PLOS ONE 10: e0122197.

Harada A, Suzuki K, Miura S, Hasui T, Kamiguchi N, Ishii T et al (2015b). Characterization of the binding properties of T-773 as a PET radioligand for phosphodiesterase 10A. Nucl Med Biol 42: 146-154.

Jäger R, Russwurm C, Schwede F, Genieser HG, Koesling D, Russwurm M (2012). Activation of PDE10 and PDE11 phosphodiesterases. J Biol Chem 287: 1210-1219.

Janavs JL, Aminoff MJ (1998). Dystonia and chorea in acquired systemic disorders. J Neurol Neurosurg Psychiatry 65: 436-445.

Jones CA, Watson DJ, Fone KC (2011). Animal models of schizophrenia. Br J Pharmacol 164: 1162-1194.

Kapur S, Mamo D (2003). Half a century of antipsychotics and still a central role for dopamine D2 receptors. Prog Neuropsychopharmacol Biol Psychiatry 27: 1081-1090.

Kapur S, Remington G, Jones C, Wilson A, DaSilva J, Houle S et al (1996). High levels of dopamine D2 receptor occupancy with lowdose haloperidol treatment: a PET study. Am J Psychiatry 153: 948-950.

Kunitomo J, Yoshikawa M, Fushimi M, Kawada A, Quinn JF, Oki H et al (2014). Discovery of 1-[2-fluoro-4-(1H-pyrazol-1-yl)phenyl]5-methoxy-3-(1-phenyl-1H-pyrazol-5-yl)pyri dazin-4(1H)-one (TAK-063), a highly potent, selective, and orally active 
phosphodiesterase 10A (PDE10A) inhibitor. J Med Chem 57: 9627-9643.

Mailly P, Charpier S, Menetrey A, Deniau JM (2003). Threedimensional organization of the recurrent axon collateral network of the substantia nigra pars reticulata neurons in the rat. J Neurosci 23: 5247-5257.

Megens AA, Hendrickx HM, Hens KA, Fonteyn I, Langlois X, Lenaerts I et al (2014). Pharmacology of JNJ-42314415, a centrally active phosphodiesterase 10A (PDE10A) inhibitor: a comparison of PDE10A inhibitors with D2 receptor blockers as potential antipsychotic drugs. J Pharmacol Exp Ther 349: 138-154.

Nishi A, Kuroiwa M, Miller DB, O'Callaghan JP, Bateup HS, Shuto $\mathrm{T}$ et al (2008). Distinct roles of PDE4 and PDE10A in the regulation of cAMP/PKA signaling in the striatum. J Neurosci 28 : 10460-10471.

Nishi A, Snyder GL, Greengard P (1997). Bidirectional regulation of DARPP-32 phosphorylation by dopamine. J Neurosci 17: 8147-8155.

Nordstrom AL, Farde L, Wiesel FA, Forslund K, Pauli S, Halldin C et al (1993). Central D2-dopamine receptor occupancy in relation to antipsychotic drug effects: a double-blind PET study of schizophrenic patients. Biol Psychiatry 33: 227-235.

Obeso JA, Rodriguez-Oroz M, Marin C, Alonso F, Zamarbide I, Lanciego JL et al (2004). The origin of motor fluctuations in Parkinson's disease: importance of dopaminergic innervation and basal ganglia circuits. Neurology 62: S17-S30.

Ouagazzal AM, Jenck F, Moreau JL (2001). Drug-induced potentiation of prepulse inhibition of acoustic startle reflex in mice: a model for detecting antipsychotic activity? Psychopharmacology (Berl) 156: 273-283.

Pani L, Pira L, Marchese G (2007). Antipsychotic efficacy: relationship to optimal D2-receptor occupancy. Eur Psychiatry 22: 267-275.

Paylor R, Crawley JN (1997). Inbred strain differences in prepulse inhibition of the mouse startle response. Psychopharmacology (Berl) 132: 169-180.

Podda MV, Riccardi E, D'Ascenzo M, Azzena GB, Grassi C (2010). Dopamine D1-like receptor activation depolarizes medium spiny neurons of the mouse nucleus accumbens by inhibiting inwardly rectifying $\mathrm{K}+$ currents through a cAMP-dependent protein kinase A-independent mechanism. Neuroscience 167: 678-690.

Russwurm C, Koesling D, Russwurm M (2015). Phosphodiesterase $10 \mathrm{~A}$ is tethered to a synaptic signaling complex in striatum. J Biol Chem 290: 11936-11947.

Sano H, Nagai Y, Miyakawa T, Shigemoto R, Yokoi M (2008). Increased social interaction in mice deficient of the striatal medium spiny neuron-specific phosphodiesterase 10A2. J Neurochem 105: 546-556.
Schmidt CJ, Chapin DS, Cianfrogna J, Corman ML, Hajos M, Harms JF et al (2008). Preclinical characterization of selective phosphodiesterase 10A inhibitors: a new therapeutic approach to the treatment of schizophrenia. J Pharmacol Exp Ther 325: 681-690.

Shirley E (1977). A non-parametric equivalent of Williams' test for contrasting increasing dose levels of a treatment. Biometrics 33: 386-389.

Simpson JN, McGinty JF (1995). Forskolin induces preproenkephalin and preprodynorphin mRNA in rat striatum as demonstrated by in situ hybridization histochemistry. Synapse 19: 151-159.

Smith SM, Uslaner JM, Cox CD, Huszar SL, Cannon CE, Vardigan JD et al (2013). The novel phosphodiesterase 10A inhibitor THPP-1 has antipsychotic-like effects in rat and improves cognition in rat and rhesus monkey. Neuropharmacology 64: $215-223$.

Stoof JC, Kebabian JW (1981). Opposing roles for D-1 and D-2 dopamine receptors in efflux of cyclic AMP from rat neostriatum. Nature 294: 366-368.

Suzuki K, Harada A, Shiraishi E, Kimura H (2015). In vivo pharmacological characterization of TAK-063, a potent and selective phosphodiesterase $10 \mathrm{~A}$ inhibitor with antipsychoticlike activity in rodents. J Pharmacol Exp Ther 352: 471-479.

Verhoest PR, Chapin DS, Corman M, Fonseca K, Harms JF, Hou X et al (2009). Discovery of a novel class of phosphodiesterase $10 \mathrm{~A}$ inhibitors and identification of clinical candidate 2-[4-(1-methyl-4-pyridin-4-yl-1H-pyrazol-3-yl)-phenoxymethyl]quinoline (PF-2545920) for the treatment of schizophrenia. J Med Chem 52: 5188-5196.

Walters DE, Howard SG (1990). The D1 agonist SKF 38393 increases dopamine release in the developing rat striatum. Eur $J$ Pharmacol 184: 257-264.

Wang A, Dorso C, Kopcho L, Locke G, Langish R, Harstad E et al (2012). Potency, selectivity and prolonged binding of saxagliptin to DPP4: maintenance of DPP4 inhibition by saxagliptin in vitro and ex vivo when compared to a rapidly-dissociating DPP4 inhibitor. BMC Pharmacol 12: 2.

Williams DA (1971). A test for differences between treatment means when several dose levels are compared with a zero dose control. Biometrics 27: 103-117.

Xie Z, Adamowicz WO, Eldred WD, Jakowski AB, Kleiman RJ, Morton DG et al (2006). Cellular and subcellular localization of PDE10A, a striatum-enriched phosphodiesterase. Neuroscience 139: 597-607.

Yoshikawa M, Kamisaki H, Kunitomo J, Oki H, Kokubo H, Suzuki A et al (2015). Design and synthesis of a novel 2-oxindole scaffold as a highly potent and brain-penetrant phosphodiesterase 10A inhibitor. Bioorg Med Chem 23: 7138-7149.

Supplementary Information accompanies the paper on the Neuropsychopharmacology website (http://www.nature.com/npp) 